\title{
El perfil docente constructor de paz: concepciones emergentes desde la escuela en el posconflicto colombiano*
}

\author{
[Versión en Castellano] \\ The Peacebuilding Teacher Profile: Emerging Concepts from the \\ School in the Colombian Post-conflict
}

O perfil do professor construtor de paz: concepções emergentes da escola no pós-conflito colombiano

Recibido el 29 de junio, 20. Aceptado el 20 de octubre, 2020.

\author{
Jorge-Mario Ortega-Iglesias** \\ http://orcid.org/0000-0002-6458-3650 \\ Colombia \\ Víctor-Enrique Valencia-Espejo*** \\ http://orcid.org/0000-0002-2838-1218 \\ Colombia
}

, Para citar este artículo:

Ortega-Iglesias, Jorge-Mario; Valencia-Espejo, Víctor-Enrique

(2021). El perfil docente

constructor de paz: concepciones emergentes desde la escuela en

el posconflicto colombiano.

Ánfora, 28(50), 103-132.

https://doi.org/10.30854/anf.v28.n50.2021.723

Universidad Autónoma de

Manizales. ISSN 0121-6538.

E-ISSN 2248-6941.

CCBY-NC-SA 4.0

\section{Resumen}

Objetivo: caracterizar las concepciones del perfil docente constructor de paz de estudiantes y profesores en escuelas públicas ubicadas en zonas de

\footnotetext{
* El artículo presenta los resultados del proyecto de investigación avalado por la Vicerrectoría de Investigación de la Universidad del Magdalena con código VIN2019124: "Concepciones del perfil docente constructor de paz: Las voces de estudiantes y profesores pertenecientes a escuelas públicas ubicadas en la Zona Troncal del Caribe en la ciudad de Santa Marta", desarrollado desde Agosto del 2018 hasta Febrero del 2020 por integrantes del Grupo de Investigación en Currículum y Evaluación GICE. Los autores declaran que no hubo conflicto de intereses en la ejecución del proyecto de investigación
}

\footnotetext{
** Licenciado en Educación Básica con énfasis en Informática, Doctor en Educación, Universidad del Magdalena, Santa Marta, Colombia. Correo electrónico: jortegai@unimagdalena.edu.co

*** Ingeniero industrial, Especialista en estadística aplicada, Universidad del Magdalena, Santa Marta, Colombia. Correo electrónico: victorvalenciaee@unimagdalena.edu.co
} 
posconflicto colombiano. Metodología: Se contempló un diseño de investigación no experimental, descriptivo, de corte transversal, mediante el uso de la técnica de Redes Semánticas Naturales Modificadas (RSNM). En el estudio participaron 233 estudiantes y 36 profesores seleccionados a partir de un muestreo no probabilístico por conveniencia, quienes asisten a cuatro instituciones educativas oficiales del sector rural (zona priorizada por el posconflicto) localizadas en la zona troncal del caribe de la ciudad de Santa Marta, en el departamento del Magdalena. Para el análisis y procesamiento de los datos se tuvieron en cuenta las categorías de la técnica RSNM: Tamaño de la Red (J), Frecuencia (F), Peso Semántico (M), Grupo SAM, Distancia Semántica (FMG) y Densidad de la Red (G); complementadas por el método Importancia Frecuencia (IF) y el análisis consensual de las RSNM configuradas por los diferentes grupos poblacionales estudiados. Resultados: las definidoras Respetuoso y Tolerante se evidenciaron como los conceptos nucleares constitutivos de la concepción del perfil docente constructor de paz desde las voces de estudiantes y profesores pertenecientes a estas escuelas. Conclusiones: la concepción del perfil docente constructor de paz en voces de estudiantes y profesores de escuelas públicas del caribe colombiano resalta valores universales y morales como el respeto y la tolerancia cuando se piensa en procesos configurativos de paz en poblaciones que históricamente han sido afectadas por el conflicto armado.

Palabras-clave: Paz; Educación para la paz; Escuela pública; Cultura de paz.

\section{Abstract}

Objective: to characterize the conceptions of the peacebuilding teacher profile of students and teachers in public schools located in Colombian post-conflict regions. Methodology: a non-experimental, descriptive, cross-sectional research design was considered, using the Modified Natural Semantic Networks (RSNM) technique. Two hundred thirty-three students and 36 teachers selected from a non-probabilistic convenience sampling participated in the study. They attend four official educational institutions in the rural sector (a zone prioritized by the post-conflict) located in the Troncal del Caribe zone of the city of Santa Marta, in the department of Magdalena. For the analysis and processing of the data, the RSNM technique categories were taken into account: Network Size (J), Frequency (F), Semantic Weight (M), SAM Group, Semantic Distance (FMG) and Density of the Network (G); they were complemented by the Importance Frequency (IF) method and the consensual analysis of the RSNM configured by the different population groups studied. Results: the defining characteristics Respectful and Tolerant were evidenced as the constitutive core concepts of the conception of the peacebuilding teacher profile from the voices of 
students and teachers belonging to these schools. Conclusions: the conception of the peacebuilding teacher profile in the voices of students and teachers of public schools in the Colombian Caribbean highlights universal and moral values such as respect and tolerance when thinking about configuring peace processes in populations that have historically been affected by the armed conflict.

Keywords: Peace; Education for peace; Public school; Peace culture.

\section{Resumo}

Objetivo: caracterizar as concepções do perfil de professor construtor de paz de alunos e professores em escolas públicas localizadas em áreas de pós-conflito colombiano. Metodologia: um projeto de pesquisa não experimental, descritivo e transversal foi contemplado através do uso da técnica de Redes Semánticas Naturais Modificadas (RSNM). O estudo envolveu 233 alunos e 36 professores selecionados a partir de uma amostragem não probabilística por conveniência, que atendem quatro instituições de ensino oficiais do setor rural (área prioritária pré-conflito) localizadas na área tronco do Caribe da cidade de Santa Marta, no departamento de Magdalena. Para a análise e processamento dos dados, foram tidas em conta as categorias da técnica RSNM: Tamanho da rede (J), Frequência (F), Peso Semântico (M), Grupo SAM, Distância Semântica (FMG) e Densidade de Rede (G); complementadas pelo método de Importância da Frequência (IF) e pela análise consensual das RSNM configuradas pelos diferentes grupos populacionais estudados. Resultados: os definidores Respetuoso e Tolerante foram evidenciados como os conceitos nucleares que constituem a concepção do perfil de ensino de construção da paz a partir das vozes de alunos e professores pertencentes a essas escolas. Conclusões: a concepção do perfil de professor construtor de paz nas vozes de estudantes e professores de escolas públicas do Caribe colombiano, destaca valores universais e morais, como respeito e tolerância ao pensar em definir processos de paz em populações historicamente afetadas por conflitos armados.

Palavras-chave: Paz; Educação para a paz; Escola pública; Cultura de paz. 


\section{Introducción}

Durante los últimos 60 años Colombia ha experimentado hechos históricos marcados por la violencia (Centro de Investigación en Relaciones Internacionales [CIDOB], 2019) con una huella de dolor y tristeza en la memoria colectiva de millones de personas que han sufrido en carne propia el conflicto armado interno.

Sin embargo, recientemente se dio un paso importante en la construcción y transición hacia condiciones de paz y justicia social, con el cese de uno de los conflictos de mayor trascendencia e historia en el país. Después de tantos años de violencia se firmó e inició la implementación de un acuerdo de paz entre el Gobierno colombiano y las Fuerzas Armadas Revolucionarias de Colombia (FARC), que trajo una oportunidad para transformar los territorios, generando más esperanza y menos violencia en las comunidades afectadas por la confrontación con este grupo armado.

Ahora, el departamento del Magdalena y su distrito capital Santa Marta, localizados en la costa norte colombiana, no han sido ajenos a los hechos de violencia generados por el conflicto armado que ha enfrentado el país a lo largo de su historia. La ubicación geográfica de este departamento ha dado lugar a numerosas confrontaciones entre diferentes grupos al margen de la ley, debido a su condición de corredor vial que comunica las fronteras de países como Venezuela, Panamá y, consecuentemente, su directa comunicación con el mar caribe y el océano pacífico (Salazar, 2016); es este un punto de preferencia geoestratégica para los actores armados.

Así, por una de sus vías principales, como lo es la zona troncal del caribe, han transcurrido actividades como la producción y transporte de cultivos de uso ilícito que han convertido a esta zona del departamento en el epicentro de disputas por obtener el control de este territorio (Quinche, Perdomo y Vargas, 2018). No obstante, la firma del acuerdo de paz con las FARC, ha posibilitado la creación de un marco jurídico y legislativo como los documentos CONPES 3850 y 3932 y el Decreto 893 del 2017 (Departamento Nacional de Planeación [DNP], 2015, 2017a, 2017b, 2018), que promueven la focalización de territorios de paz y la creación de programas y proyectos que apuntan al fortalecimiento de los centros educativos como espacios protectores promotores de paz y la reconciliación, espacios que focalizan al departamento del Magdalena y su distrito capital Santa Marta como zonas priorizadas por el posconflicto.

Según Tassara (2017) el reto para Colombia consiste en vigilar el cumplimiento de estos acuerdos y encaminarse en un proceso de construcción de paz y desarrollo sostenible, en el que el Gobierno Nacional debe hacer frente para mejorar las causas políticas, sociales y económicas que generaron inicialmente 
el conflicto, "empezando por el fortalecimiento del Estado, la lucha contra la pobreza y la desigualdad, la promoción de la inclusión social, el acceso a la tierra de los campesinos pobres y el impulso al desarrollo rural" (Tassara, 2017, p. 455). En este sentido, el Gobierno colombiano es consciente de que la construcción de paz, además del fin de un conflicto armado, tiene como objetivo auspiciar la disminución de las desigualdades sociales, económicas, territoriales y de acceso a la justicia, a las que se ven enfrentados principalmente los habitantes de las regiones más afectadas por su incidencia (DNP, 2018).

Entonces, se ha desarrollado un Plan Marco de Implementación que "orienta las políticas públicas requeridas para el cumplimiento del acuerdo de paz durante los próximos 15 años y facilita el seguimiento necesario por parte de las diferentes estancias dispuestas para este fin" (DNP, 2018, p.11). No obstante, este tipo de procesos puede tardar y requerir la inversión de muchos recursos en programas de recuperación y rehabilitación en la población afectada (Lappin, 2018; Jones y Metzger, 2018; Baumgart-Ochse, 2014), que incluye tanto a las víctimas del Estado como a las víctimas de los grupos subversivos.

Estas posibilidades pensadas para todos los actores del conflicto han llevado a que los acuerdos de paz entre el gobierno y las FARC, a pesar de haber generado grandes expectativas en el pueblo colombiano, susciten diversos enfrentamientos de carácter político y social evidenciado en fuertes posturas tanto de apoyo como de oposición al proceso de paz.

Por esta razón, la academia y las demás comunidades que trabajan alrededor de la paz y el posconflicto colombiano deben mantener un rol activo y conciliador, que propicien la generación de espacios para estudiar y comprender las particularidades de este conflicto armado y sus repercusiones, los beneficios de convivir en una sociedad pacífica y las posibilidades para aportar a la construcción de paz desde los diversos entornos y posiciones en que se desenvuelven los ciudadanos.

Una mejor comprensión del conflicto y de sus consecuencias acerca más a la paz (Muñoz, 2004; Hernández, Luna y Cadena, 2017), por lo cual, "educar en y para el conflicto resulta ser un reto de la educación para la paz que se concreta en: descubrir la perspectiva positiva del conflicto" (Caireta y Barbeito, 2005, p. 23), "aprender a analizar los conflictos, descubrir su complejidad (...) y encontrar soluciones que permitan afrontar los conflictos sin violencia” (Cascón, 2001, p. 5).

La educación para la paz se constituye, entonces, como una potente estrategia para la construcción social de la paz en las comunidades y territorios afectados por la violencia históricamente (Acevedo y Báez, 2018), su implementación implica, entre otros aspectos, una transformación paradigmática de la escuela que privilegie el valor por la vida y fomente la formación de ciudadanos íntegros con múltiples valores, que contribuyan a la búsqueda de una justicia social, la 
convivencia con los mínimos de dignidad y calidad de vida a partir del desarrollo de sus cualidades como personas y el compartir en comunidad, promoviendo una forma de vida que invite a disfrutar de la belleza de vivir (Lira y Archivaldo, 2014; Garzón, 2017).

El Estado colombiano, asume la paz según el Artículo 22 de la Constitución Política de 1991 y la educación de acuerdo con el Artículo 67 (Presidencia de la República, 1991), como derechos fundamentales; de esta forma, se manifiesta el compromiso social de fomentar y propiciar en los niños, jóvenes y adolescentes afectados por el conflicto, el desarrollo de competencias, actitudes y valores que conduzcan a la reconstrucción social y convivencia pacífica en las comunidades.

En esta dirección, y en el marco de la configuración de nuevos escenarios promotores de paz en los territorios, el Gobierno colombiano, a partir del Decreto 1038 del Ministerio de Educación Nacional, MEN (2015) asume la cátedra de la paz como una materia independiente y de obligatoriedad en todas las instituciones educativas de preescolar, básica y media para las escuelas públicas y privadas; se entiende la cátedra de la paz como una estrategia que busca enseñar a los jóvenes desde las escuelas a convivir en armonía, respetar las diferencias y resolver conflictos de manera pacífica en sus entornos.

Educar para la paz comprende un esfuerzo colectivo orientado a la reconstrucción del tejido social y emocional devastado en estas comunidades, donde las escuelas a partir de un enfoque humanístico promuevan un nuevo desarrollo cultural generando propuestas alternativas, diversas e incluyentes para aprender a ser y a ejercer la ciudadanía de forma responsable y consciente. En este marco de implementación, la responsabilidad de educar para la paz en las escuelas propone como protagonista al profesor que comparte día a día con los estudiantes y los padres de familia.

De esta manera, las concepciones y el papel de un profesor que construye paz a partir de sus acciones pedagógicas en el aula y en la comunidad, son fundamentales para cumplir con el propósito de formar ciudadanos capaces de convivir pacíficamente en una sociedad. En consecuencia, los conocimientos que requieren los docentes constructores de paz, más que conceptos teóricos, refieren al hecho de generar conciencia sobre sus propios límites e "interiorizar valores como el respeto, la solidaridad, la cooperación o el trabajo en equipo” (Mora, 2016, p. 78).

Los desafíos que asume el profesorado como actor clave de paz en los territorios y la escuela invitan a

Seguir cuestionando con profunda reflexión sobre: ¿quién es el profesor que educa en nuestras escuelas?, ¿cuáles son sus necesidades de formación?, ¿están preparados 
nuestros maestros para contribuir con la formación de sujetos de manera coherente y acertada ante las necesidades de su entorno? (Ortega, 2018, p. 8).

Estos cuestionamientos precisan un espacio oportuno para indagar sobre el perfil del profesor que aporta a la construcción de paz en el territorio colombiano, principalmente desde el sistema de concepciones que los miembros de la comunidad educativa han podido configurar alrededor del profesor constructor de paz. Las concepciones son comprendidas como un "paraguas mental", es decir, como una "estructura mental general, que incluye las creencias, los significados, conceptos, las proposiciones, reglas, imágenes mentales, preferencias y gustos" (Thompson, 1992, p. 130), cuyos constructos actúan como verdaderos marcos y principios de actuación de los sujetos, permeando en el caso de los profesores, sus modelos didácticos, incluso orientando tanto o más su práctica que aquellos principios científicos que soportan la profesión y el saber disciplinar (Pardo, 2006; Ortega, 2012, 2019).

Para efectos de este estudio, se abordan las concepciones sobre el reconocimiento de constructos cognitivos y sociales, es decir, palabras, términos y demás expresiones que guardan una representación puntual y relación histórica sobre el perfil del maestro que construye paz; todos ello, desde las voces de actores escolares que han vivido el conflicto de manera directa.

Los profesores cuentan con amplias posibilidades para mediar en los principales sujetos sociales de las nuevas generaciones que participan en el entorno educativo, particularmente en los estudiantes, padres de familia, comunidad e incluso sus compañeros docentes (Lira y Archivaldo, 2014); por tal razón, están llamados a liderar la iniciativa en la estrategia de educación para la paz. En este sentido, el profesor como constructor de paz en el aula y en la comunidad, debe asumir un rol activo para la formación en valores de los niños, que les permita aprender a convivir y tener la capacidad de desarrollar procesos de reconciliación, arrepentimiento y negociación, para solucionar los conflictos a los cuales se enfrenten de una manera pacífica (Hernández et al., 2017).

En este orden de ideas, el presente estudio se pregunta por ¿Cuáles son las concepciones sobre el perfil docente constructor de paz que tienen los estudiantes y profesores de escuelas públicas ubicadas en la Zona Troncal del Caribe en la ciudad de Santa Marta?, escuelas que, para el caso del caribe colombiano, han vivido el conflicto de una manera distinta. Así pues, educar para la paz y la orientación de la cátedra para la paz en estas poblaciones merece el reconocimiento del sistema de concepciones sobre el perfil del docente constructor de paz inmerso en estas realidades locales y situadas, para apostar a un proceso de construción con sentido y valor para las personas que habitan en las comunidades y son parte de este ecosistema escolar. 
La idea de caracterizar las concepciones sobre el perfil del profesor que construye paz en esta zona de posconflicto colombiano, representa un aporte hacia la generación de una cultura de paz en estas localidades, fundamentalmente desde el escenario escolar, una paz que no puede ser pensada desde lugares foráneos sino desde las mismas voces de los actores y victimas del conflicto.

Ahora, la paz es un concepto que ha evolucionado a través de la historía; hoy, su comprensión ha trascendido de la ausencia de la guerra, la violencia y los conflictos armado hacia una necesidad imprescindible para ejercer y disfrutar los derechos humanos, partiendo de la exigencia de la justicia social entre las sociedades, y reconociendo la igualdad y la dignidad de todos los pueblos y las culturas (Arango, 2007; Fisas, 2011; Harto de Vera, 2017). En este sentido, la paz es considerada un valor universal añorado por la comunidad y que representa el bienestar personal, grupal y de especie que nos permite sentirnos más humanos y darle sentido a nuestras vidas (Bouché, 2012; Molina y Muñoz, 2004).

Sin embargo, la construcción de la paz no es una tarea fácil para Colombia, este proceso ha singnificado una tarea de alta complejidad, puesto que su implementación, implica, entre otros elementos de alto valor social y político, trabajar por el perdón y reconciliación entre los actores armados del conflicto, las víctimas y la ciudadanía en general, promoviendo una cultura de paz para transformar esa cultura de violencia tan presente en nuestras sociedades (Fisas, 2011). Generar una cultura de paz es "un proceso de transformación individual, colectiva e institucional que surge de las creencias y acciones de los propios individuos y evoluciona (...) dentro de su propio contex to histórico, sociocultural y económico" (Organización de las Naciones Unidas para la Educación, la Ciencia [UNESCO], 1998, p. 4), por lo cual, caracterizar las concepciones de estudiantes y profesores de escuelas ubicadas en entornos afectados históricamente por el conflicto armado, resulta fundamental para comprender como se entiende y se aporta desde la perspectiva de la función social del docente a la construcción de paz en los territorios. 


\section{Metodología}

Este estudio se desarrolló a partir de un diseño de investigación no experimental, descriptivo de corte transversal, con la participación de un grupo de 233 estudiantes y 36 profesores de los grados noveno, décimo y undécimo de cuatro escuelas ubicadas en la Zona Troncal del Caribe de la ciudad de Santa Marta. La selección de los participantes en el estudio se realizó a partir de un muestreo no probabilístico por conveniencia (Otzen y Manterola, 2017) donde se definió como criterio de inclusión (Ávila, 2006) su disponibilidad, receptividad y aprobación.

Para aproximarnos a la concepción que tienen estudiantes y profesores de estas escuelas manifiesta en constructos cognitivos (expresiones, palabras, conceptos) se usó la técnica de Redes Semánticas (Hickman, et al., 2016), y se empleó como dispositivo de captura de datos la técnica de Redes Semánticas Naturales Modificadas (RSNM) (Figueroa, González y Solís, 1981). Esta técnica se considera como una reconstrucción en forma gráfica y matemática de la estructura mental de un sujeto o colectivo (Allan, 2008) definida como el conjunto de palabras recuperadas a partir de las "ideas y conceptos que las personas construyen acerca de cualquier objeto, tema o situación de su cotidianidad y que son expresados en términos coloquiales" (Vargas-Garduño, Méndez-Puga y Vargas-Silva, 2014, p. 5).

Así mismo, a partir de esta técnica fue posible establecer objetivamente el significado natural que los sujetos otorgan a una idea, concepto o constructo en función de su propia experiencia (Figueroa, González y Solís, 1981; Reyes, 1993; Valdez, 2000), permitiendo la recuperación histórica de los significados que estudiantes y profesores de estas escuelas han configurado sobre perfil del docente constructor de paz.

El proceso de recolección de datos contempló el diseño y aplicación de un cuestionario a estudiantes y profesores, para indagar sobre aquellas características que definen el perfil del docente constructor de paz. Para esto, se formuló la pregunta estímulo ¿Cuáles son las características que definen a un profesor constructor de paz? y se solicitó a los participantes relacionar un listado de 10 palabras (sustantivos, adjetivos y verbos) que describen a un profesor que construye paz en su escuela.

Finalmente, se solicitó que jerarquizaran las palabras listadas, otorgando el número 1 a aquella definidora que tiene una relación más fuerte con la pregunta estímulo, y el número 10, a aquella definidora que presenta una relación más débil. Este cuestionario se aplicó de forma grupal y se desarrolló en un espacio de 5 minutos. Todos los participantes conocieron previamente el propósito del proyecto, así como también los aspectos éticos para el tratamiento de los datos, firmando el correspondiente consentimiento informado. 
El análisis de los datos contempló las categorías de la técnica RSNM sugeridas por Figueroa, González y Solís (1981), Reyes (1993), Valdez (2000) y Valdez, González y Sánchez (2005):

- Tamaño de la red (J): Representa el número total de palabras utilizadas por los sujetos de investigación para describir el perfil del docente constructor de paz.

- Frecuencia $(\mathrm{F})$ : Indica el número de veces que es mencionada cada palabra para describir el concepto estímulo.

- Peso semántico (M): Representa la relevancia significativa que le otorga un grupo poblacional a cada definidora y se obtiene a partir de la sumatoria de los valores jerárquicos atribuidos por los individuos a cada palabra.

- Grupo SAM: Conjunto de definidoras que presentan el mayor peso semántico dentro de la red configurada por el grupo poblacional en estudio. Se logra visualizar cuando se grafican los pesos semánticos de forma descendente en un diagrama de barras y se identifica el lugar en que la curva tiende a ser asintótica con relación al eje de las abscisas.

- Distancia semántica (FMG): Indicador expresado en porcentaje (RSN) o en valores numéricos entre o y 1 (RSNM) que permite identificar que tan cercana o distante se encuentra una definidora del núcleo de la red. En RSN se obtiene al dividir el peso semántico de cada palabra del grupo SAM, entre el peso semántico de la definidora con mayor relevancia significativa para los sujetos de investigación. En RSNM, por su parte, se obtiene al calcular la diferencia entre la distancia semántica (RSN) de la definidora mejor ponderada con respecto a la distancia semántica (RSN) de cada palabra del grupo SAM.

- Densidad $(\mathrm{G})$ : Indicador que expresa el grado de cercanía o dispersión existente entre las palabras que conforman el grupo SAM y se obtiene al calcular el promedio de las diferencias entre las distancias semánticas de las palabras contiguas pertenecientes al grupo SAM.

Además, entendiendo que el método de Importancia Frecuencia (IF) y la técnica Redes Semánticas Naturales Modificadas (RSNM) son coincidentes y complementarios, se apeló a la relación de estas herramientas metodológicas para el 
fortalecimiento del análisis de los datos y el robustecimiento de las conclusiones del proyecto (González et al., 2018). Así, relacionar el estudio de RSNM con el método IF permitió clasificar las definidoras que conforman el grupo SAM de acuerdo con su frecuencia y grado de importancia en cuadrantes denominados: zona central, zonas de cambio potencial y zona periférica (Dany, Lo Monaco y Urdapilleta, 2015) (Tabla 1). La zona central, se compone por el grupo de definidoras que presentaron una frecuencia y peso semántico alto, es decir, aquellas palabras que los sujetos de investigación relacionaron con mayor fuerza con el perfil del docente constructor de paz.

En contraste, la zona periférica la componen aquellas definidoras que presentaron una frecuencia y peso semántico bajo, es decir, aquellas palabras que con el paso del tiempo los sujetos de investigación pueden dejar de relacionar con el constructo estímulo. Finalmente, considerando que la relación de las RSNM y el método IF nos permite comparar la constitución de las definidoras entre distintos grupos poblacionales (González, et al., 2018) en este estudio se analizaron a partir de una matriz de consensos la relación, similitudes y diferencias encontradas entre las RSNM configuradas por estudiantes, profesores y el total de participantes.

Tabla 1. Método IF

\begin{tabular}{c|c|c|} 
Importancia & Alta & Baja \\
\hline Frecuencia & $\begin{array}{c}\text { Zona central o Núcleo de la red: } \\
\text { Definidoras con importancia } \\
\text { alta y frecuencia de aparición } \\
\text { alta. }\end{array}$ & $\begin{array}{c}\text { Zona de cambio potencial o } \\
\text { elementos contrastantes: De- } \\
\text { finidoras con importancia baja } \\
\text { y frecuencia de aparición alta. }\end{array}$ \\
\hline Baja & $\begin{array}{c}\text { Zona de cambio potencial o pri- } \\
\text { mera periferia: Definidoras con } \\
\text { importancia alta y frecuencia de } \\
\text { aparición baja. }\end{array}$ & $\begin{array}{c}\text { Zona periférica o de segunda } \\
\text { periferia: Definidoras con } \\
\text { importancia baja y frecuencia } \\
\text { de aparición baja. }\end{array}$ \\
\hline
\end{tabular}

Fuente: adaptado de Dany et al. (2015)

En el método IF, el valor que determina si una definidora presenta una frecuencia alta o baja es la frecuencia promedio del grupo SAM; es así como aquella definidora que presente una frecuencia inferior a la frecuencia promedio del grupo SAM es considerada "definidora con frecuencia baja" y aquella que presente una frecuencia superior al promedio es considerada "definidora con frecuencia alta”. Así mismo, el valor que determina si una definidora presenta un grado de 
importancia alto o bajo es la distancia semántica promedio (RSN), considerando de esta manera como "definidora con importancia alta” a aquellas palabras con una distancia semántica superior al promedio, y como "definidora con importancia baja” a aquellas palabras con una distancia semántica inferior al promedio.

\section{Resultados}

\section{Concepciones de los estudiantes}

Los estudiantes configuraron una RSNM de tamaño $\mathrm{J}=124$, relacionando 124 palabras con perfil del docente constructor de paz. El conjunto de definidoras que presentan la mayor relevancia significativa (grupo SAM) para los estudiantes se relacionan en la siguiente tabla:

Tabla 2. Grupo SAM de la RSNM configurada por estudiantes

\begin{tabular}{|c|c|c|c|c|c|c|}
\hline & No. & Definidoras & Frecuencia & $\begin{array}{l}\text { Peso semántico } \\
\text { (M) }\end{array}$ & $\begin{array}{r}\text { Distanci } \\
(F\end{array}$ & ántica \\
\hline & 1 & Respetuoso & 189 & 1429 & $100,00 \%$ & 0 \\
\hline & 2 & Responsable & 106 & 807 & $56,50 \%$ & 0,44 \\
\hline & 3 & Tolerante & 126 & 770 & $53,90 \%$ & 0,46 \\
\hline & 4 & Honesto & 95 & 601 & $42,10 \%$ & 0,58 \\
\hline & 5 & Paciente & 98 & 599 & $41,90 \%$ & 0,58 \\
\hline 芯 & 6 & Amable & 97 & 590 & $41,30 \%$ & 0,59 \\
\hline$\frac{2}{2}$ & 7 & Amigable & 113 & 565 & $39,50 \%$ & 0,6 \\
\hline & 8 & Comprensivo & 73 & 423 & $29,60 \%$ & 0,7 \\
\hline & 9 & Amoroso & 60 & 346 & $24,20 \%$ & 0,76 \\
\hline & 10 & Confiable & 45 & 279 & $19,50 \%$ & 0,8 \\
\hline & 11 & Humilde & 41 & 248 & $17,40 \%$ & 0,83 \\
\hline & 12 & Cariñoso & 52 & 234 & $16,40 \%$ & 0,84 \\
\hline
\end{tabular}

Fuente: elaboración propia

Respetuoso es la definidora que presenta la mayor frecuencia de aparición (F) y relevancia significativa $(\mathrm{M})$, mostrando una distancia semántica (FMG) importante con respecto a las otras definidoras pertenecientes al grupo SAM; 
así, el respeto es considerado por los estudiantes como un valor universal trascendente para la promoción de una cultura de paz en las escuelas (Arango, 2007) y como una característica fundamental al momento de definir el perfil del profesor que construye paz en estos entornos.

Esto implica que, un profesor constructor de paz debe preocuparse principalmente por mantener relaciones interpersonales con sus estudiantes en la que prime el respeto y el buen trato, sin ejercer una relación de autoridad de dominio-sumisión sobre ellos (Sánchez, 2011). Adicionalmente, se destaca que en esta RSNM definidoras como Responsable, Tolerante, Honesto, Paciente, Amable, Amigable, Comprensivo, Amoroso, Confiable, Humilde y Cariñoso, también cuentan con una frecuencia de aparición y peso semántico importante, por lo que resulta significativo su consideración para entender en un sentido más amplio la concepción que estos sujetos manifiestan. En la figura 1 se aprecia la distancia semántica existente entre el conjunto de definidoras que configuran el grupo SAM.

Figura 1. Distancia semántica entre las definidoras relacionadas por estudiantes

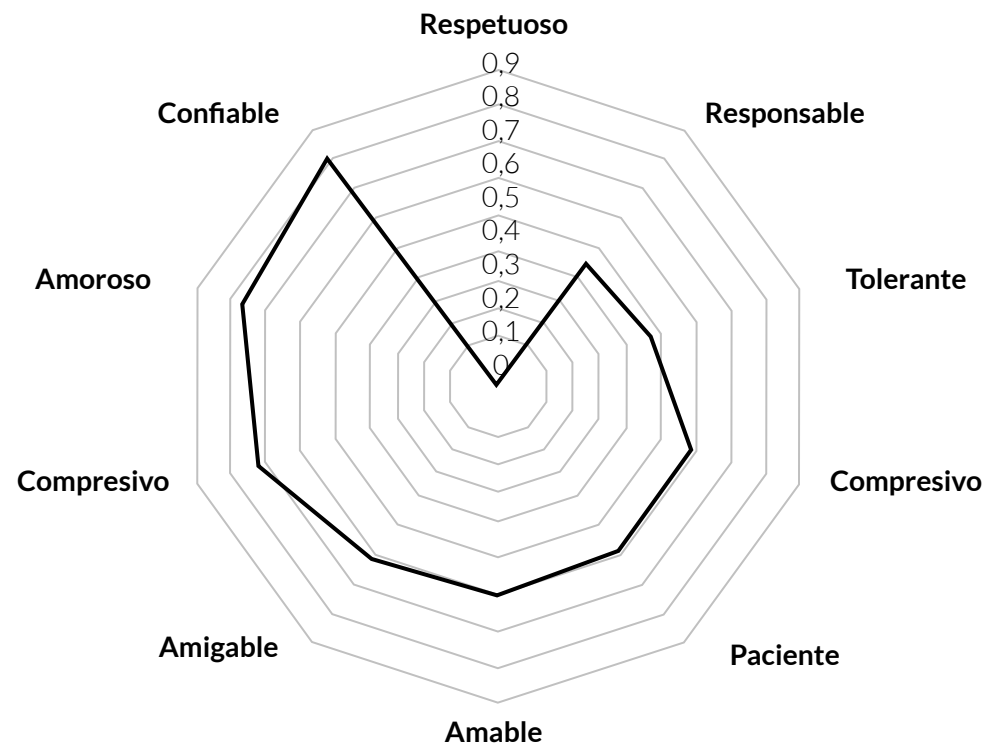

Fuente: elaboración propia 
Por presentar el mayor peso semántico (M) Respetuoso se ubica en el núcleo de la red, las definidoras Responsable, Tolerante, Honesto, Paciente, Amable, Amigable, Comprensivo, Amoroso, Confiable, Humilde y Cariñoso se distancian del núcleo de forma proporcional con la disminución del grado de relevancia significativa otorgado. El análisis de la distancia semántica entre las palabras contenidas en la figura 1 permiten evidenciar que esta RSNM presentó una densidad de la red $(\mathrm{G})$ correspondiente a un 7,6\%. La aplicación del método IF permitió identificar el grupo de palabras que se ubican en la zona central de la red, las zonas de cambio potencial y la zona de periferia (Tabla 3).

Tabla 3. Clasificación por importancia y frecuencia del grupo SAM (estudiantes)

\begin{tabular}{|c|c|c|}
\hline Importancia & \multirow{2}{*}{ Alta } & \multirow{2}{*}{ Baja } \\
\hline Frecuencia & & \\
\hline Alta & $\begin{array}{c}\text { Respetuoso }(F=189, M=1429, \\
F M G=100 \%) \\
\text { Tolerante }(F=126, M=770, \\
F M G=54 \%) \\
\text { Honesto }(F=95, M=601, F M G=42 \%) \\
\text { Paciente }(F=98, M=599, F M G=42 \%) \\
\text { Amable }(F=97, M=590, F M G=41 \%) \\
\text { Amigable }(F=113, M=565, F M G=40 \%)\end{array}$ & \\
\hline Baja & & $\begin{array}{c}\text { Comprensivo }(F=73, M=423, \\
\text { FMG }=30 \%) \\
\text { Amoroso }(F=60, M=346, \\
\text { FMG }=24 \%) \\
\text { Confiable }(F=45, M=279, \\
\text { FMG }=20 \%) \\
\text { Humilde }(F=41, M=248, \\
\text { FMG }=17 \%) \\
\text { Cariñoso }(F=52, M=234, \\
\text { FMG }=16 \%) \\
\text { Sincero }(F=33, M=195, \\
F M G=14 \%)\end{array}$ \\
\hline
\end{tabular}

Fuente: elaboración propia

Se observa que las definidoras Respetuoso, Tolerante, Honesto, Paciente, Amable y Amigable configuran la zona central o núcleo de la RSNM de los estudiantes, indicando su relativa estabilidad en la memoria colectiva de los sujetos con el paso del tiempo. En el caso de la zona de periferia, se localizaron las definidoras Comprensivo, Amoroso, Confiable, Humilde, Cariñoso y Sincero. 
Los resultados asociados a esta RSNM destacan un comportamiento particular, observándose la ausencia de definidoras ubicadas en las zonas de cambio potencial en la matriz del método IF. Se aprecia que en el conjunto de palabras mencionadas el mayor número de veces siempre presentó un grado de relevancia significativo alto y las definidoras mencionadas pocas veces, siempre presentaron un grado de relevancia significativa bajo.

Además, esta RSNM presenta la menor densidad $(G)$ entre los grupos de análisis estudiados, lo que sugiere, que el grupo de estudiantes presenta una concepción homogénea, estable, coherente y que naturalmente se relaciona con la memoria colectiva y la historia de vida de los sujetos (González, et al., 2018). Se exalta el respeto, la tolerancia, la honestidad, la paciencia, la amabilidad y la amigabilidad como las características definidoras del sujeto profesor que construye paz.

\section{Concepciones de los profesores}

La RSNM configurada por los profesores permitió identificar un conjunto de 71 características asociadas con la concepción del perfil docente constructor de paz, de las cuales, 10 conforman el grupo SAM (ver Tabla 4).

Tabla 4. Grupo SAM de la RSNM configurada por profesores

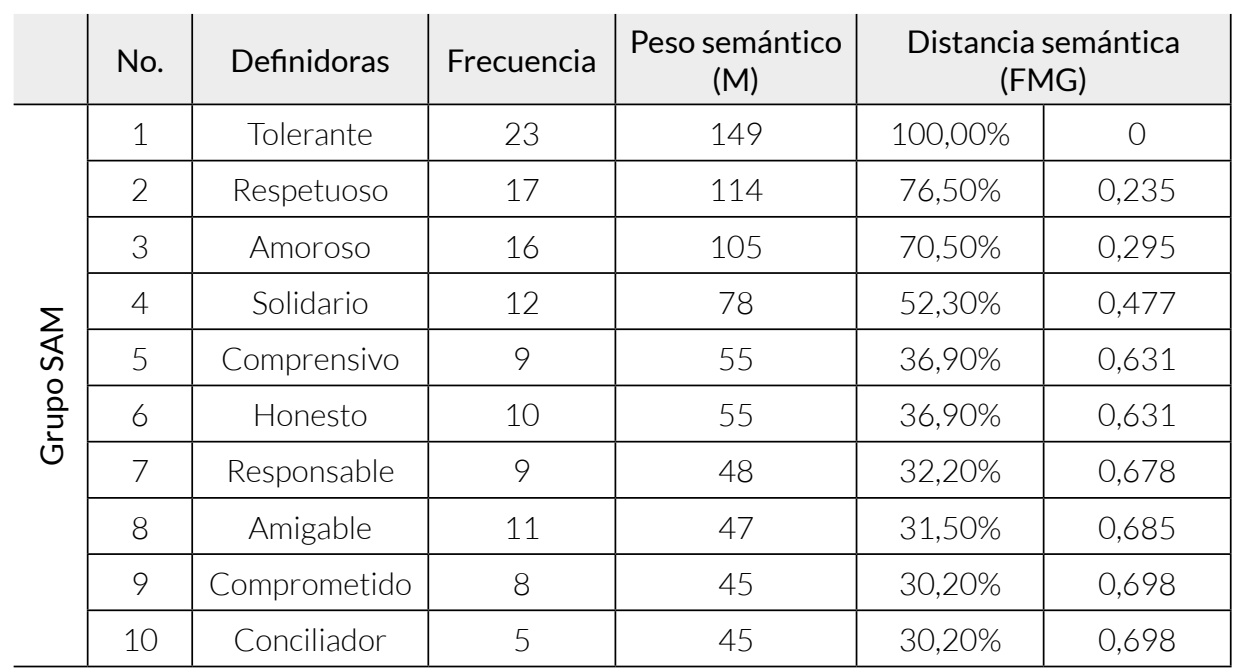

Fuente: elaboración propia 
Las definidoras más relevantes que manifiestan los profesores son: Tolerante, Respetuoso, Amoroso, Solidario, Comprensivo, Honesto, Responsable, Amigable, Comprometido y Conciliador, entre estas, se destaca la Tolerancia, que presenta una mayor relevancia significativa y frecuencia de aparición. La Tolerancia, al igual que el Respeto (en el caso de los estudiantes), corresponde a un valor moral que evidencia la capacidad de las personas para valorarse como iguales, hecho que se asume como la base ética de la paz (Reardon, 1999). La identificación de la Tolerancia en esta RSNM ayuda a comprender la necesidad manifiesta por estos sujetos de ser reconocidos y valorados como iguales, en un ambiente que históricamente ha promovido su victimización, abonado especialmente por el abandono estatal y la violencia generada por los grupos armados que tienen presencia en dicha zona. La figura 2 muestra de forma gráfica la distancia existente entre las palabras que conforman el grupo SAM de esta RSNM.

Figura 2. Distancia semántica entre las definidoras relacionadas por los profesores

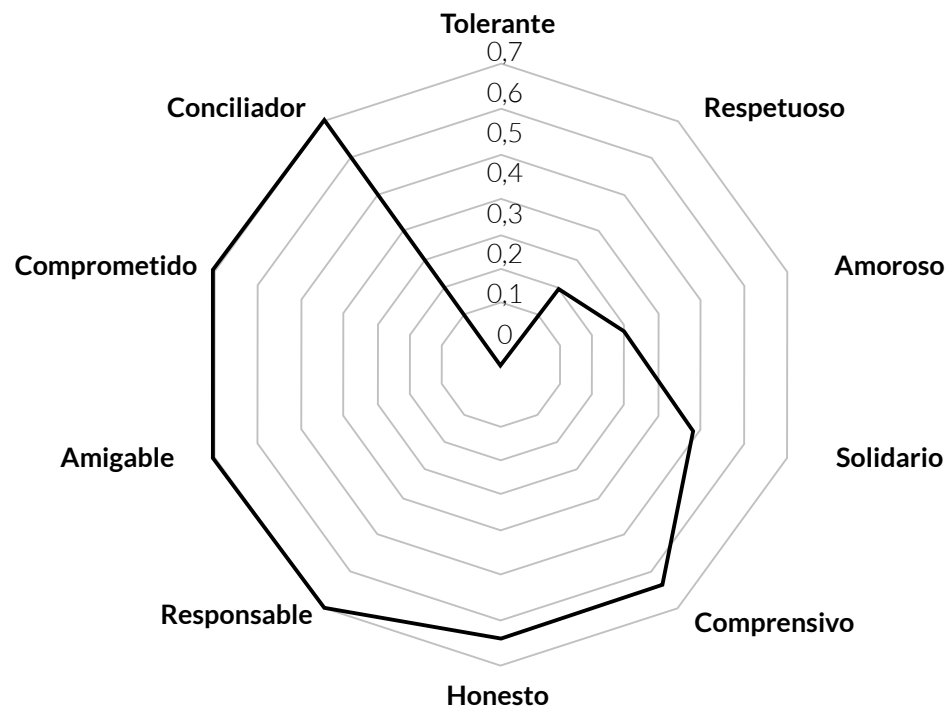

Fuente: elaboración propia

La palabra Tolerante se ubica en la zona central de la red como la definidora que presenta el mayor grado de importancia $(\mathrm{M})$ y frecuencia de aparición $(\mathrm{F})$ para los profesores, así como su conformación del núcleo central de la RSNM. En cuanto a la distancia semántica correspondiente a cada palabra de este grupo 
SAM, se pudo determinar que la densidad de la red $(\mathrm{G})$ es de $7,76 \%$. En cuanto a los resultados provenientes del método IF, se observa la siguiente clasificación (taba 5).

Tabla 5. Clasificación por importancia y frecuencia del grupo SAM (profesores)

\begin{tabular}{|c|c|c|}
\hline Importancia & \multirow{2}{*}{ Alta } & \multirow{2}{*}{ Baja } \\
\hline Frecuencia & & \\
\hline Alta & $\begin{array}{c}\text { Tolerante }(F=23, M=149, \\
F M G=100 \%) \\
\text { Respetuoso }(F=17, M=114, \\
F M G=77 \%) \\
\text { Amoroso }(F=16, M=105, F M G=70 \%) \\
\text { Solidario }(F=12, M=78, F M G=52 \%)\end{array}$ & \\
\hline Baja & & $\begin{array}{c}\text { Comprensivo }(F=9, M=55, \\
\text { FMG }=37 \%) \\
\text { Honesto }(F=10, M=55, \\
F M G=37 \%) \\
\text { Amigable }(F=11, M=47, \\
F M G=32 \%) \\
\text { Conciliador }(F=5, M=45, \\
F M G=30 \%) \\
\text { Pacífico }(F=6, M=44, F M G=30 \%) \\
\text { Paciente }(F=8, M=38, F M G=26 \%)\end{array}$ \\
\hline
\end{tabular}

Fuente: elaboración propia

Las definidoras Tolerante, Respetuoso, Amoroso y Solidario hacen parte de la zona central de esta red. Así mismo, en la zona de periferia se encuentran ubicadas las definidoras Comprensivo, Honesto, Amigable, Conciliador, Pacífico y Paciente, las cuales, a pesar de ser reiterativas en la red, no representan una relevancia significativa y frecuencia de aparición alta. Se destaca, al igual que en el caso de los estudiantes, la RSNM configurada por los profesores no presenta definidoras ubicadas en las zonas de cambio potencial, lo que sugiere una concepción homogénea y estable sobre el perfil docente constructor de paz. 


\section{Concepciones de estudiantes y profesores}

Estudiantes y profesores relacionaron en conjunto 297 características (RSNM de tamaño $\mathrm{J}=297$ ) con el perfil del docente constructor de paz. En la Tabla 6 se puede apreciar el grupo SAM de esta RSNM.

Tabla 6. Grupo SAM de la RSNM configurada por estudiantes y profesores

\begin{tabular}{|c|c|c|c|c|c|c|}
\hline & No. & Definidoras & Frecuencia & $\begin{array}{c}\text { Peso semántico } \\
(\mathrm{M})\end{array}$ & Distanc & lántica \\
\hline \multirow{10}{*}{ 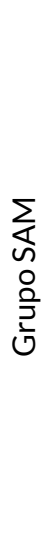 } & 1 & Respetuoso & 206 & 1543 & $100 \%$ & 0 \\
\hline & 2 & Tolerante & 149 & 919 & $60 \%$ & 0,4 \\
\hline & 3 & Responsable & 115 & 855 & $55 \%$ & 0,45 \\
\hline & 4 & Honesto & 105 & 656 & $43 \%$ & 0,57 \\
\hline & 5 & Paciente & 106 & 637 & $41 \%$ & 0,59 \\
\hline & 6 & Amable & 104 & 620 & $40 \%$ & 0,6 \\
\hline & 7 & Amigable & 124 & 612 & $40 \%$ & 0,6 \\
\hline & 8 & Comprensivo & 82 & 478 & $31 \%$ & 0,69 \\
\hline & 9 & Amoroso & 76 & 451 & $29 \%$ & 0,71 \\
\hline & 10 & Confiable & 48 & 289 & $19 \%$ & 0,81 \\
\hline
\end{tabular}

Fuente: elaboración propia

Se destacan las definidoras Respetuoso, Tolerante, Responsable, Honesto, Paciente, Amable, Amigable, Comprensivo, Amoroso y Confiable. Entre este conjunto de palabras, la definidora Respetuoso presenta el mayor peso semántico $(\mathrm{M})$, frecuencia de aparición $(\mathrm{F})$ y una distancia semántica $(\mathrm{FMG})$ significativa con respecto a las demás definidoras. En la figura 3 podemos apreciar de manera gráfica la distancia semántica existente entre cada una de las definidoras que conforman el grupo SAM de esta RSNM. 
Figura 3. Distancia semántica entre las definidoras relacionadas por estudiantes y profesores

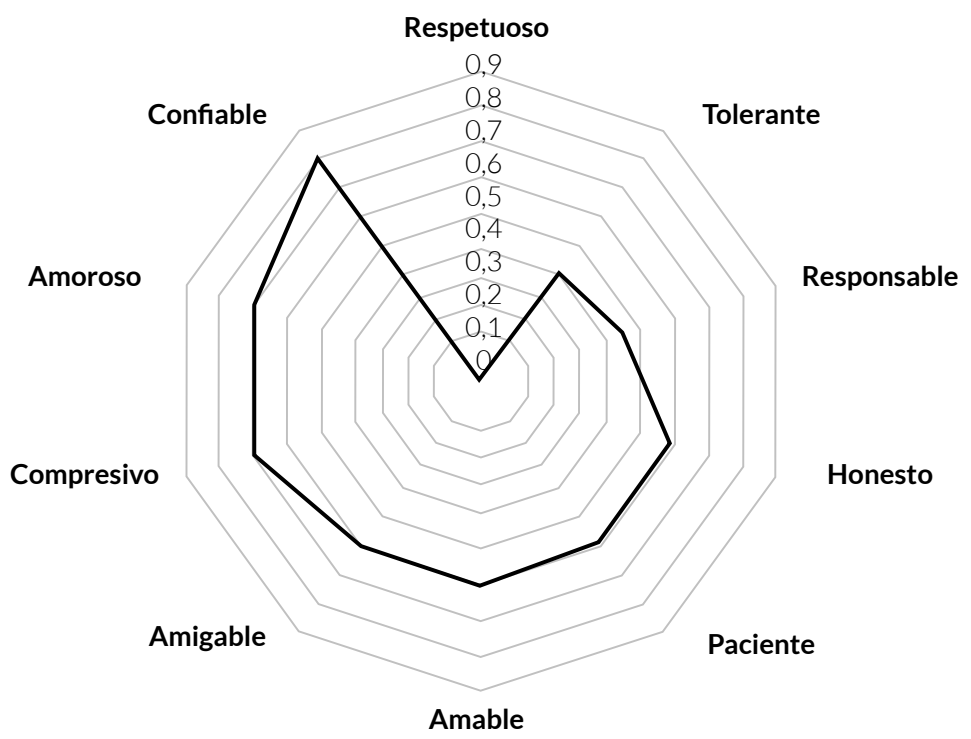

Fuente: elaboración propia

Así, la palabra Respetuoso se ubica en el núcleo de la red presentando el mayor grado de relevancia significativa. Esta RSNM presenta la mayor densidad G $=9,03 \%$ entre las redes analizadas, evidenciando una mayor dispersión entre los grados de importancia otorgados a cada una de las definidoras pertenecientes al grupo SAM. De acuerdo con el método IF, las definidoras en esta red se clasifican como se observa en la Tabla 7.

Tabla 7. Clasificación por importancia y frecuencia del grupo SAM (estudiantes y profesores)

\begin{tabular}{c|c|c|} 
Importancia & Alta & Baja \\
\cline { 1 - 1 } Frecuencia & Respetuoso $(F=206, M=1543$, & Paciente $(F=106, M=637$, \\
\cline { 1 - 2 } Alta & $F M G=100 \%)$ & $F M G=41 \%)$ \\
& Tolerante $(F=149, M=919$, & Amigable $(F=124, M=612$, \\
& $F M G=60 \%)$ & $F M G=40 \%)$ \\
\hline
\end{tabular}




\begin{tabular}{c|c|c|} 
Importancia & Alta & Baja \\
\cline { 1 - 1 } Frecuencia & & Amable $(F=104, M=620$, \\
& & $F M G=40 \%)$ \\
& & Comprensivo $(F=82, M=478$, \\
& & $F M G=31 \%)$ \\
& Honesto $(F=105, M=656$, & Amoroso $(F=76, M=451$, \\
& $F M G=43 \%)$ & $F M G=29 \%)$ \\
& & Confiable $(F=48, M=289$, \\
& & SMG $=19 \%)$ \\
& & Solidario $(F=50, M=266$, \\
& $F M G=17 \%)$ \\
\hline
\end{tabular}

Fuente: elaboración propia

El núcleo de la red o zona central indica que las definidoras Respetuoso y Tolerante son las más representativas. También se puede apreciar que en las zonas de cambio potencial se ubican las definidoras Honesto, Paciente y Amigable y en la zona de periferia se ubican las definidoras Amable, Comprensivo, Amoroso, Confiable y Solidario. Lo anterior supone una estrecha relación entre la definición de valores universales y morales y la concepción del perfil docente constructor de paz.

La concepción del perfil docente constructor de paz manifestada por estudiantes y profesores privilegia valores universales y morales como el respeto y la tolerancia cuando se construye paz en entornos que históricamente han sido afectados por el conflicto. Tal condición brinda pistas para comprender la función social que el entorno escolar podría ofrecer, como medio transformador y constructor de paz; así, la escuela, bajo el liderazgo visible de los profesores, está llamada a fomentar una educación integral que promueva principalmente la formación en valores, constituyéndose en posibles bases éticas, pedagógicas y metodológicas para la efectiva construcción de una paz estable y duradera.

\section{Consenso entre estudiantes y profesores}

En la tabla 8 se muestra la matriz de consensos de las RSNM configuradas por los tres grupos poblacionales en estudio. 
Tabla 8. Matriz de consensos entre grupos poblacionales

\begin{tabular}{|c|c|c|c|} 
Grupo poblacional & Estudiantes & Profesores & Total \\
\hline \multirow{5}{*}{ Estudiantes } & Respetuoso & & \\
& Tolerante & & \\
& Honesto & & \\
& Paciente & & \\
& Amable & & \\
& Amigable & & \\
\hline \multirow{3}{*}{ Profesores } & & Respetuoso & \\
& Respetuoso & Tolerante & \\
& Tolerante & Amoroso & \\
& & Solidario & \\
\hline \multirow{2}{*}{ Total } & Respetuoso & Respetuoso & Respetuoso \\
& Tolerante & Tolerante & Tolerante \\
\hline
\end{tabular}

Fuente: elaboración propia

La diagonal de la matriz muestra las definidoras que pertenecen a la zona central de las RSNM configuradas por cada grupo poblacional (tablas 3,5 y 7 ) y las celdas inferiores dan cuenta de las coincidencias entre las concepciones de estos grupos poblacionales, donde se puede identificar que existe un consenso generalizado entre estudiantes, profesores y participantes en general, al relacionar las definidoras Respetuoso y Tolerantes con su concepción del perfil del docente constructor de paz en sus escuelas.

\section{Conclusiones}

La comprensión del concepto de paz, como un derecho humano de tercera generación de carácter colectivo (Arango, 2007) que se asocia además de la ausencia de guerra, al desarrollo de la justicia social, la igualdad y el diálogo, permitió el surgimiento de la investigación para la paz como un campo interdisciplinario que hoy en día convoca a un gran número de académicos, investigadores y activistas del mundo. Así, desde la investigación para la paz se plantea que la capacidad de las personas para construir paz cuando cooperan, se reconocen como iguales y crean vínculos afectivos (Muñoz y Molina, 2010); vínculos en los cuales valores como la equidad, la tolerancia, la solidaridad, la justicia, el diálogo, la libertad, la democracia y el respeto cumplen un rol protagónico al desarrollar procesos de construcción de paz (Cabello et al., 2016; Rodríguez e Hinojosa, 2017). 
En este orden de ideas, es necesario dar sentido y valor a la configuración del perfil docente constructor de paz, siendo coherentes y exaltando las realidades locales y situadas de territorios afectados por el conflicto armado; por eso, desde este estudio, se pregunta por las concepciones que tienen estudiantes y profesores sobre el perfil docente constructor de paz en escuelas públicas ubicadas en la zona troncal del caribe colombiano.

La caracterización de las concepciones de estudiantes y profesores sobre el perfil del docente constructor de paz coinciden al señalar Respetuoso (mencionada por el $76 \%$ de los participantes) y Tolerante (mencionada por el $55 \%$ de los participantes) como las características clave que definen al sujeto profesor, principalmente desde su intencionalidad en los procesos de interacción pedagógica para la formación de ciudadanos y la configuración de escenarios de paz en las escuelas y el territorio. Esta lectura coincide con los planteamientos de Rodríguez e Hinojosa (2017) al destacar la importancia de valores como el respeto y la tolerancia en el proceso de promoción y adquisición de la cultura de paz en los diversos territorios. No obstante, según la UNESCO (2017) las concepciones identificadas en estos grupos poblacionales sobre las competencias y perfil del rol del maestro frente a los procesos de construcción de paz pueden verse de corto alcance, dado que se otorga poca relevancia a principios básicos como la libertad, la justicia, la democracia, los derechos humanos y la solidaridad, elementos clave para la construcción de una cultura de paz a nivel universal.

La cultura de paz consta de valores, actitudes, comportamientos, conocimientos y habilidades que ayudan a la resolución pacífica de los conflictos y a la creación de las condiciones necesarias para la construcción de paz (UNESCO, 2017; Fondo de las Naciones Unidas para la Infancia [UNICEF], 2015), en contraste, las concepciones de estudiantes y profesores evidenciadas en este estudio, privilegian valores puntuales (Respeto y Tolerancia) y dejan de considerar factores como los conocimientos y habilidades propias que debe tener un profesor que construye paz. Este tipo de consideraciones proponen lecturas alternativas para la formación y desarrollo profesional del profesorado, dado que visibilizan competencias fundamentales que requieren los maestros para trabajar en comunidades afectadas por el conflicto armado. Así pues, la formulación de dichas competencias merece una lectura que pase por las realidades y necesidades de las comunidades y territorios que han sido víctimas del conflicto. De igual forma, la emergencia de dicho perfil del profesor constructor de paz instala un debate muy interesante sobre: ¿qué tipo de sujeto queremos formar en estas comunidades?, ¿cuáles son las competencias que deben adquirir estos sujetos?, ¿cómo serán sus procesos de evaluación y en qué medida esta evaluación aporta al crecimiento y desarrollo de sus comunidades? 
El hecho de que estudiantes y profesores ponderen el reconocimiento de valores como el Respeto y la Tolerancia, antes que conocimientos y habilidades específicas de los docentes, atiende a los cuestionamientos existentes en la literatura sobre la formación docente en el campo actitudinal y conceptual para afrontar la educación para la paz y la interculturalidad, debido a que estas nociones no son tenidas en cuenta durante el desarrollo de las prácticas educativas y los saberes docentes (Torres, 2019). Ante esto, es importante considerar el papel que tienen las experiencias de vida de los miembros de la comunidad educativa que participaron de este proyecto frente al conflicto armado, puesto que son actores escolares afectados históricamente por situaciones de violencia, comportamientos agresivos y de permanente rivalidad, en donde, además, "es habitual detectar la fórmula de dominio-sumisión entre unos 'iguales' que acaban siendo desiguales por la fuerza de la inercia” (Mateo et al., 2004, p. 9).

Por otro lado, al analizar las RSNM configuradas por los dos grupos poblacionales de manera independiente (estudiantes y profesores), se identificaron que las definidoras Honesto, Paciente, Amable y Amigable son características que los estudiantes consideran importantes para la definición del perfil docente constructor de paz; sin embargo, los profesores no presentan esta misma concepción y, en lugar de ello, destacan la importancia de las definidoras Amoroso y Solidario. De esta manera, se evidencia que existen diferencias relativas entre el núcleo de las RSNM configuradas por los grupos poblacionales contrastados (González et al., 2018), cuyos hallazgos plantean la necesidad de una visión complementaria y enriquecida a la hora de identificar el conjunto de características que constituyen al profesor constructor de paz en estos escenarios.

Estas diferencias evidenciadas en el estudio confluyen en un gran reto que invita a desarrollar procesos investigativos que indaguen sobre el sentido histórico que estudiantes y profesores de estas instituciones educativas atribuyen a conceptos como la honestidad, la paciencia, la amabilidad, la amigabilidad, el amor y la solidaridad. Lo anterior, es indispensable para poder comprender los significados relacionados con las concepciones del perfil docente constructor de paz a partir de la elaboración de significados con mayor especificidad que tengan en cuenta la riqueza cultural, histórica y contextual de esta comunidad, permitiendo de esta manera analizar cuál es la percepción de la realidad que tienen los miembros de estas comunidades educativas y cuál es la interpretación propia que le otorgan al concepto de paz (Álvarez y Pérez, 2019).

Ahora, también es importante mencionar algunas limitaciones que a futuro permitan mejorar el proceso investigativo y la caracterización de este perfil profesional, por ejemplo: se requiere indagar las concepciones de otros actores pertenecientes a la comunidad educativa como padres de familia, líderes sociales e incluso actores en calidad de victimarios. La configuración de la red semántica 
en tal caso podría ser mucho más completa e incluiría una mayor diversidad de conceptos y definidoras que robustecerían la red y su núcleo constitutivo. Adicionalmente, es conveniente complementar este tipo de estudios a partir de diseños metodológicos que permitan la recuperación de las voces, relatos y demás componentes del orden discursivo configurativo de estas poblaciones.

Estos elementos aquí expuestos aproximan a una caracterización de las concepciones del perfil docente constructor de paz, de una manera local y situada, que brindan hallazgos importantes para que, mediante investigaciones, sean analizados, debatidos y reflexionados con mayor profundidad. De igual forma, existen otros aspectos relacionados y de igual importancia que son fundamentales para construir masa crítica y avanzar en nuevas investigaciones que aborden pistas de aspectos formativos necesarios para la reconstrucción del tejido social en estos lugares, entre ellos, se requiere seguir cuestionándose por aspectos tales como: ¿por qué los sujetos de estas comunidades consideran que el respeto y la tolerancia son las características principales que definen al profesor constructor de paz?, ¿qué significado en su historia de vida tiene para los estudiantes y profesores de estas escuelas el respeto y la tolerancia? Tales cuestionamientos son una suerte de antesala que pone de manifiesto la capacidad de reinventarnos como sociedad, especialmente desde la escuela como escenario configurador y promotor de ciudadanos.

\section{Referencias}

Acevedo, A.; Báez, A. (2018). La educación en cultura de paz. Herramienta de construcción de paz en el posconflicto. Reflexión Política, 20(40), 69-80. https://revistas.unab.edu.co/index.php/reflexion/article/ view/3455/2931

Allan, V. (2008). Medir el significado: las redes semánticas como método de investigación sociológica (Tesis inédita). Universidad de Chile.

Álvarez, A.; Pérez, C. (2019). Educación para la paz: aproximación teórica desde los imaginarios de paz. Educación y Educadores, 22(2) 277-296. http:// www.scielo.org.co/pdf/eded/v22n2/0123-1294-eded-22-02-277.pdf

Arango, V. (2007). Paz social y cultura de paz. Panamá Viejo. 
Ávila, H. (2006). Introducción a la metodología de la investigación. Instituto Tecnológico de CD.

Baumgart-Ochse, C. (2014). Opposed or Intertwined? Religious and Secular Conceptions of National Identityin Israeland the Israeli-Palestinian Conflict. Politics, Religion \& Ideology, 15(3), 40 1-420. https:// www.tandfonline.com/ $\mathrm{doi} /$ full $/ 10.1080 / 21567689.2014 .948531$ ? scroll=top\&needAccess $=$ true

Bouché, J. (2012). La paz comienza por uno mismo. Educación XXI, 6(1). 25-43. http://revistas.uned.es/index.php/educacionXX1/article/ view/351/304.

Cabello, P.; Carmona, S.; Gorjón, F.; Iglesias, E.; Sáenz, K.; Vásquez, R. (2016). Cultura de paz. Grupo Editorial Patria, S.A. de C.V.

Caireta, M.; Barbeito, C. (2005). Introducción de conceptos: paz, violencia, conflicto. Escola de Cultura de Pau.

Cascón, P. (2001). Cátedra Unesco sobre paz y derechos humanos: Educar en y para el conflicto. Universidad Autónoma de Barcelona.

Centro de investigación en relaciones internacionales, CIDOB. (2019). Conflicto en Colombia: antecedentes históricos y actores. https://www.cidob.org/ publicaciones/documentacion/dossiers/dossier_proceso_de_paz_en_ colombia/dossier_proceso_de_paz_en_colombia/conflicto_en_colombia_ antecedentes_historicos_y_actores

Dany, L.; Lo Mónaco, G.; Urdapilleta, I. (2015). Free associations and social representations: Some reflections on rankfrequency and importancefrequency methods. Quality \& Quanty International Journal of Methodology, 49(2), 489-507. https://www.researchgate.net/publication/272202641_ Free_associations_and_social_representations_Some_reflections_on_ rank-frequency_and_importance-frequency_method

Departamento Nacional de Planeación, DNP. (2015). Fondo Colombia en Paz. Documento CONPES 3850. https://colaboracion.dnp.gov.co/CDT/ Conpes/Econ\%C3\%B3micos/3850.pdf 
Departamento Nacional de Planeación, DNP. (2017a). Plan Marco de Implementación: Acuerdo final para la terminación del conflicto y la construcción de una paz estable y duradera.https://colaboracion.dnp.gov.co/CDT/ Poltica\%20de\%20Vctimas/PAZ/3932_Anexo\%20B_Plan\%20Marco\%20 de\%20Implementaci\%C3\%B3n\%2O(PMI).pdf

Departamento NacionaldePlaneación.(2017b).DocumentosCONPES3850.https:// colaboracion.dnp.gov.co/CDT/Conpes/Econ\%C3\%B3micos/3850.pdf

Departamento Nacional de Planeación. (2018). Lineamientos para la articulación del plan marco de implementación del acuerdo final con los instrumentos de planeación, programación y seguimiento a políticas públicas de orden nacional y territorial (Documento CONPES 3932). Bogotá D.C., Colombia: DNP. Figueroa, J.; González, E.;

Fisas, V. (2011). Educar para una cultura de paz. Quaderns de Construcción de Pau, 20. https://escolapau.uab.cat/img/qcp/educar_cultura_paz.pdf

Fondo de las Naciones Unidas para la Infancia, UNICEF.(2015). Research Cosortium on Education and Peacebuilding. Literature Review: The Role of Teachers in Peacebuilding. Amsterdam: UNICEF. https://educationanddevelopment. files.wordpress.com/2014/11/the-role-of-teachers-in-peacebuilding-ata-glance-nov 15.pdf

Garzón, F. (2017). ¿Educación para la paz? Revista Educación y desarrollo social, $11(2)$, 5-19. https://revistas.unimilitar.edu.co/index.php/reds/article/ view/3249

González, A.; García, T.; Soltero, R.; Correa, F.; Reynoso, O. (2018). Una reflexión metodológica sobre el método de importancia frecuencia y las redes semánticas naturales en el estudio de las representaciones sociales. Revista de Educación y Desarrollo, 46(3), 23-32. http://www.smip.udg.mx/ sites/default/files/10._una_reflexion_metodologica_sobre_el_metodo_ importancia-frecuencia_y_las_redes_semanticas.pdf

Harto de Vera, F. (2017). La construcción del concepto de paz: Paz negativa, Paz positiva y Paz imperfecta. Cuadernos de estrategia, 183, 119-146. https://dialnet.unirioja.es/servlet/articulo? codigo $=5832796$ 
Hernández, I.; Luna, J.; Cadena, M. (2017). Cultura de paz: una construcción desde la educación. Revista Historia de la Educación Latinoamericana, 19(28), 149-172. http://www.scielo.org.co/scielo.php?script=sci_ abstract\&pid=So 122-72382017000100009\&lng=en\&nrm=iso\&tlng=es

Hickman, H.; Alarcón, M.; Cepeda, M.; Cabrera, R.; Torres, X. (2016). Significado de buen profesor y de evaluación docente por estudiantes y maestros universitarios. La técnica de redes semánticas. Sinéctica, 47. http://www. scielo.org.mx/scielo.php?script=sci_arttext\&pid=S1665-109X20160002 oooo9\&lng=es\&tlng $=$ es

Jones, B.; Metzger, S. (2018). Evaluating Conflict Dynamics: A Novel Empirical Approach to Stage Conceptions. Journal of Conflict Resolution, 62(4), 819847. https://journals.sagepub.com/doi/full/10.1177/0022002716656448

Lappin, R. (2018). Bridging the Capabilities-Expectations Gap: Lessons Learned from Pst-Conflict Democracy Assistance to Liberia. Democracy and Security, 15(3), 248-270.

Lira, Y.; Archivaldo, H. (2014). La educación para la paz como competencia docente: aportes al sistema educativo. Innovación Educativa, 14(64), 123144. http://www.scielo.org.mx/pdf/ie/v14n64/v14n64a10.pdf

Mateo, P.; Ayala, A.; Pérez, L.; Gutiérrez, R. (2004). Educar en relación: Hacia la convivencia y el respeto. Educrea.

Ministerio de Agricultura y Desarrollo Rural (2017). Decreto 893 (28 de Mayo). Por el cual se crean los Programas de Desarrollo con Enfoque Territorial-PDET. http://es.presidencia.gov.co/normativa/normativa/DECRETO\%20 893\%20DEL\%2028\%20DE\%20MAYO\%20DE\%202017.pdf. Bogotá: MADR.

Ministerio de Educación Nacional Colombia, MEN. (2015). Decreto 1038 (25 de Mayo). Por el cual se reglamenta la Cátedra de la Paz. https://www. funcionpublica.gov.co/eva/gestornormativo/norma.php?i=61735

Molina, B.; Muñoz, F. (2004). Manual de Paz y Conflictos. Instituto de la Paz y los Conflictos, Granada. 
Mora, P. (2016). Conocimientos, habilidades y actitudes de los docentes constructores de paz. Mundo Fesc, 6(11), 72-79. https://www.fesc.edu.co/ Revistas/OJS/index.php/mundofesc/article/view/84

Muñoz, F. (2004). La paz. En: Molina, B. y Muñoz, F. Manual de Paz y Conflictos (pp. 21-41). Instituto de la Paz y los Conflictos, Granada.

Muñoz, F.; Molina, B. (2010). Una cultura de paz compleja y conflictiva: La búsqueda de equilibrios dinámicos. Revista Paz y Conflictos, 3, 44-61. https://www.ugr.es/ revpaz/articulos/rpc_n3_2010_art3.pdf

Organización de las Naciones Unidas para la Educación, la Ciencia, UNESCO. (1998). Informe preliminar de síntesis a las Naciones Unidas acerca de la cultura de paz. París: UNESDOC. https://unesdoc.unesco.org/ark:/48223/ pfoooo 11 1280_spa?posInSet= 1 \&queryId =af4 18 daf-7e4b-4672-8f9ba5b92a61a9ob

Organización de las Naciones Unidas para la Educación, la Ciencia, UNESCO. (2017). Transformative pedagogy for peace-building: A guide for Teachers. https://unesdoc.unesco.org/ark:/48223/pfooo0261349

Ortega, J;; Perafán, A. (2012). Algunas tendencias en la investigación sobre el conocimiento profesional docente: antecedentes y estados de la cuestión. Revista EDUCyT, 6, 17-29. https://1library.co/document/ zlgp4eoy-tendencias-investigacion-conocimiento-profesional-docenteantecedentes-actual-cuestion.html

Ortega, J. (2018). Hacia una resignificación del perfil profesional del profesor en la escuela colombiana. Revista Praxis, 14(1), 7-8. https://revistas.unimagdalena.edu.co/index.php/praxis/article/ download/2713/2240?inline $=1$

Ortega, J. (2019). El concepto de tecnología escolar: una mirada alternativa. Editorial Unimagdalena.

Otzen, T.; Materola, C.(2017). Técnicas de muestreo sobre una población a Estudio. International Journal of Morphology, 35(1), 227-232. https://scielo.conicyt. $\mathrm{cl} /$ scielo.php?script=sci_arttext\&pid=So7 17-95022017000100037 
Pardo, A. (2006). Creencias de profesores y estudiantes acerca de la clase académica. Revista de investigación Universidad de la Salle, 6(2), 308-318. https://www.researchgate.net/publication/237043048_Creencias_de_ profesores_y_estudiantes_acerca_de_la_clase_academica

Presidencia de la República (1991). Constitución Política de Colombia. Ed. Legis.

Quinche, J.; Perdomo, P.; Vargas, J. (2018). Despojo paramilitar en el Magdalena: El papel de las élites económicas y políticas. Observatorio de Restitución y Regulación de Derechos de Propiedad Agraria, Colombia.

Reardon, B. (1999). La tolerancia: umbral para la paz. UNESCO.

Reyes, L. (1993). Las redes semánticas naturales, su conceptualización y su utilización en la construcción de instrumentos. Revista de Psicología Socialy Personalidad, 9(1), 83-99. http://www.scielo.org.co/scielo.php?script=sci_ nlinks\&ref=000 164\&pid=S1657-9267201300020001900029\&lng $=$ en

Rodríguez, K.; Hinojosa, M. (2017). Respeto y tolerancia como valores promotores de la Cultura de Paz en padres de niños en edad de preescolar en el municipio de San Nicolás de los Garza, Nuevo León México. Pensamiento Americano, 10(18), 96-114. http://publicaciones.americana. edu.co/index.php/pensamientoamericano/article/view/47

Sánchez, M. (2011). La cultura para la paz en Colombia: retos y opciones desde una perspectiva psico-jurídica. Pensamiento Jurídico, 30, 63-101. https://revistas.unal.edu.co/index.php/peju/article/view/36711

Salazar, G. (2016). El conflicto armado y su incidencia en la configuración territorial: Reflexiones desde la ciencia geográfica para la consolidación de un periodo de pos-negociación del conflicto armado en Colombia. Bitácora Urbano Territorial, 26(2), 45-57. https://revistas.unal.edu.co/index.php/ bitacora/article/view/57605

Solís, V.(1981). Una aproximación al problema del significado:las redes semánticas. Revista Latinoamericana de Psicología, 13(3), 447-458. http://www. scielo.org.co/scielo.php? script $=$ sci_nlinks\&ref $=000165 \& \mathrm{pid}=\mathrm{S} 1794-$ $9998201500010000900012 \& \operatorname{lng}=\mathrm{es}$ 
Tassara, C. (2017). Posconflicto y políticas públicas en Colombia: una mirada internacional a un reto nacional. En E. Tremolada. Desafios del multilateralismo y de la paz (pp. 453-503). Universidad Externado de Colombia.

Thompson, A. (1992). Teachers' beliefs and conceptions: A synthesis of the research. In D. Graws (Ed.). Handbook of research on mathematics teaching and learning: A Project of the National Counsil of Teachers of Mathematics (pp.127-143). Macmillan.

Torres, M. (2019). Educación para la paz y formación profesional: Aproximación desde la investigación documental. Praxis \& Saber, 1O(2), 143-167. https://revistas.uptc.edu.co/index.php/praxis_saber/article/view/7424

Valdez, J. (2000). Las redes semánticas naturales, usos y aplicaciones en psicología social. Universidad Autónoma del Estado de México.

Valdez, J.; Gonzáles, N.; Sánchez, Z. (2005). Elección de pareja en universitarios mexicanos. Enseñanza e investigación en psicología, 10(2), 355-367. https://www.redalyc.org/pdf/292/29210210.pdf

Vargas-Garduño, M.; Méndez-Puga, A.; Vargas-Silva, A. (2014). La técnica de las redes semánticas naturales modificadas y su utilidad en la investigación cualitativa. Memoria Académica IV Encuentro Latinoamericano de Metodología de las Ciencias Sociales, 27 al 29 de agosto de 2014, Heredia, Costa Rica. http://www.memoria.fahce.unlp.edu.ar/trab_eventos/ev.8204/ev.8204.pdf 